\title{
SUBDIVISION SURFACE MID-SURFACE RECONSTRUCTION OF TOPOLOGY OPTIMIZATION RESULTS AND THIN- WALLED SHAPES USING SURFACE SKELETONS
}

\author{
Denk, Martin (1); \\ Rother, Klemens (2); \\ Paetzold, Kristin (1) \\ 1: Bundeswehr University Munich, Institute for Technical Product Development; \\ 2: Munich University of Applied Sciences, Institute for Material and Building Research
}

\begin{abstract}
Polygon meshes and particularly triangulated meshes can be used to describe the shape of different types of geometry such as bicycles, bridges, or runways. In engineering, such polygon meshes can occur as finite element meshes, resulting from topology optimization or laser scanning. This article presents an automated parameterization of polygon meshes into a parametric representation using subdivision surfaces, especially in topology optimization. Therefore, we perform surface skeletonization on a volumetric grid supported by the Euclidian distance transformation and topology preserving and shape-preserving criterion. Based on that surface skeleton, an automated conversation into a Subdivision Surface Control grid is established. The final mid-surface-like parametrization is quite flexible and can be changed by variating the control gird or the local thickness.
\end{abstract}

Keywords: Computational design methods, Computer Aided Design (CAD), Lightweight design, Skeletonisation, Topology Optimization

\section{Contact:}

Denk, Martin

Bundeswehr University Munich

Insitute for Technical Product Development

Germany

martin.denk@unibw.de 


\section{INTRODUCTION}

Various applications such as animation design, topology optimization, or 3D laser scanning apply discrete triangulated meshes to represent the surface of a 3D geometry. Triangulated surfaces describe the shape of the object by separating the boundary surface into discrete triangles. Engineering applications for manufacturing, optimization, or design modification require such a parametric representation as, for example, constructive solid geometry (CSG) or free form surfaces of the geometry (Bénière et al., 2013; Vidal et al., 2014). The boundary representation of an object can be reconstructed with primitive surface types (Bénière et al., 2013; Gauthier et al., 2017; Vidal et al., 2014) or free form surfaces (Ben Makhlouf et al., 2019; Louhichi et al., 2015), referred as boundary representation surface (Brep). Primitive surface types are typically used for (CSG) (Bénière et al., 2013; Gauthier et al., 2017; Vidal et al., 2014), whereas free form surfaces are used for the reconstruction of organic geometries (Ben Makhlouf et al., 2019; Louhichi et al., 2015; Yoely et al., 2018). Particularly in Topology Optimization, such free form surfaces can directly result using isogeometric analysis (Dedè et al., 2012; Gao et al., 2020, 2019) or level set methods (Lian et al., 2017; Picelli et al., 2018) as Breps. In contrast to the Brep provided by surface fitting or optimization, the skeleton enables a powerful shape description with fewer parameters, which can be used for shape adjustment (Denk et al., 2020). Figure 1 shows an example of a curve skeleton representation covered in (Denk et al., 2020) with 12 vertices and 12 radii parameters and its similar boundary representation considering 142 vertices. Such flexibility enables a robust and fast manual modification by designers using computer graphic software or automated with shape morphing (Storti et al., 1997; Wu et al., 2016) to fulfill, for example, manufacturing constraints (Adam and Zimmer, 2015) for additive manufacturing (AM). Particular experimental parameter studies for support structures in AM require an as simple as possible shape description so that only a few experiments are necessary to cover the correlation or cross-correlation (Weber et al., 2020).
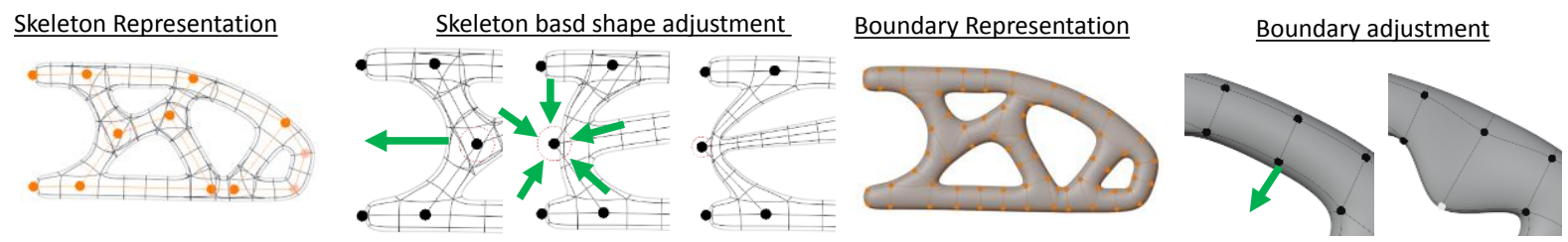

Figure 1: Shape adjustment using skeleton and boundary representation

Such skeleton-based reverse engineering can be applied for deformed FE-meshes represented in (Ben Makhlouf et al., 2019; Louhichi et al., 2015), point cloud reconstruction (Kresslein et al., 2018, p.), the determination of center lines in CT-Data (Computer Tomography) to reconstruct blood vessels (Hua Li and Yezzi, 2006) or vascular skeletons (Lidayová et al., 2016), topology optimization results provided by level set methods (Lian et al., 2017; Picelli et al., 2018) or homogenization method (Bendsøe and Sigmund, 1999; Denk et al., 2020b), by rasterization of the geometry, or directly on CT-Data.

Geometric reverse engineering attempts to redesign a parametric shape and topology of representations such as polygon meshes (Bénière et al., 2013; Gauthier et al., 2017; Vidal et al., 2014) or volumetric geometries (Bremicker et al., 1991). Most of the approaches perform a so-known mesh segmentation (Agathos et al., 2007), where the boundary of the geometry is split into several patches (Bénière et al., 2013; Gauthier et al., 2017; Vidal et al., 2014). Afterward, the surface of the geometry can be parametrized in surface types like primitive surfaces (Bénière et al., 2013; Gauthier et al., 2017; Vidal et al., 2014) or non-uniform rational B-spline (NURBS) surfaces (Ben Makhlouf et al., 2019; Louhichi et al., 2015). This segmentation only results in surface parametrization (Bénière et al., 2013; Gauthier et al., 2017; Vidal et al., 2014), so that topological properties are not covered. Therefore, the so-known curve (middle line) or surface skeleton (middle surface) can be used for part segmentation (Agathos et al., 2007; Feng et al., 2015; Reniers and Telea, 2008a), which can lead to a representation in beamline shape (Bremicker et al., 1991; Nana et al., 2017; Stangl and Wartzack, 2015). While curve skeleton can be used for part segmentation of organic shapes (Nana et al., 2017; Reniers and Telea, 2008a; Stangl and Wartzack, 2015), the surface skeleton can be used for patch segmentation (Reniers and Telea, 2008b) and part segmentation (Feng et al., 2015), or a hybrid form using surface skeleton for patch-part segmentation (Koehoorn et al., 2017). While points on a curve skeleton can be classified in view cases such as junction-, end- and skeletal point, the surface skeleton extends the number of cases for parametrization dramatically (Hisada et al., 2001; Saha et al., 2000; Svensson et al., 2002). However, 
curve skeleton recognition is mostly restricted to organic tubular-like geometries with similar circular cross-sections for reasonable shape preservation (Nana et al., 2017; Stangl and Wartzack, 2015; Tagliasacchi et al., 2016). So, for the parametrization, there is a trade-off between the number of parameters increasing using a surface skeleton and lack in shape covering while using curve skeletons. The following figure shows a concept of applying medial balls of (Blum, 1967), the 3D representation as a polygon mesh and a 3D image and its curve and surface skeleton using the thinning method of (Lee et al., 1994), which iteratively peels the voxels on the surface until the curve or surface skeleton preserves.
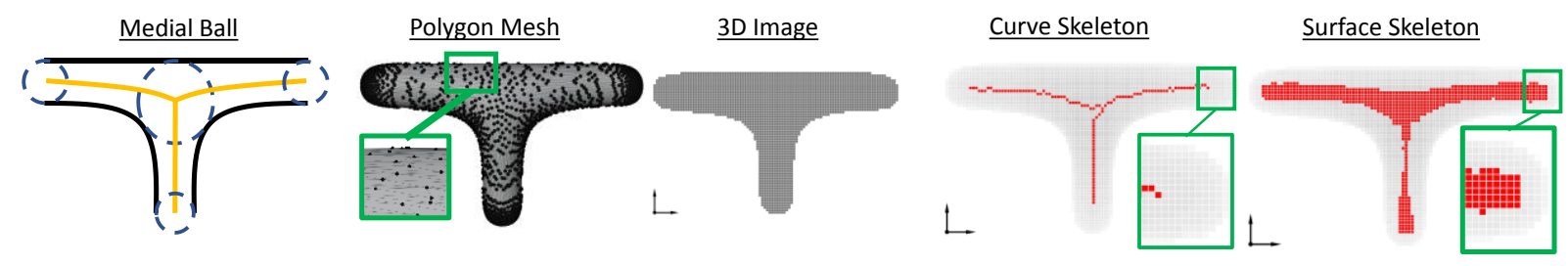

Figure 2: Concept of skeletonization for reverse engineering of polygon meshes

Our work focuses on the parametrization with Catmull-Clark subdivision surfaces of non-tubelike geometries in topology optimization and for thin-walled parts using surface skeletonization, which extends our previous work (Denk et al., 2020) focused on curve skeleton (see Figure 1). To cover the complexity of surface skeletonization, we process 1D voxel lines extracted from the surface skeleton so that only a small amount of categorization is required. Therefore, this work covers in section 3 the automated surface skeletonization and control grid estimation, which is followed by a subdivision surface parametrization. The methods are tested on topology optimized results using the homogenization method and thin-walled structures such as honeycombs or part of the airplane fuselage in section 4 .

\section{STATE OF THE ART}

A 3D segmentation for $\mathrm{CAD}$ applications can be achieved by various methods such as spectral analysis, clustering, region growing, skeleton-based methods (Agathos et al., 2007), or applying machine learning (Denk et al., 2019; Wang et al., 2018). The state of the art of this article refers to the skeleton-based methods, where first, the skeleton is extracted, and then the shape parameter such as cross-sections are determined. For other concepts, we refer to the summary of (Agathos et al., 2007).

The curve skeleton has been used in a wide range, whereas the surface skeleton has only been used sparsely (Koehoorn et al., 2017; Mayer and Wartzack, 2020; Yin et al., 2020). For beam-like shapes, first, the beamline in the form of a curve skeleton is estimated, which is followed by the determination of the cross-sections (Bremicker et al., 1991; Kresslein et al., 2018; Nana et al., 2017; Stangl and Wartzack, 2015). The reconstruction of the organic models typically follows several steps. First, a pre-processing of the geometry is performed, such as smoothing of triangulated meshes or growing of binary images (Bremicker et al., 1991). Secondly, a curve skeleton is extracted by methods such as thinning (Bremicker et al., 1991; Yin et al., 2020), Voronoi diagram (Mayer and Wartzack, 2020), or mesh contraction (Kresslein et al., 2018; Nana et al., 2017; Stangl and Wartzack, 2015). Thirdly, a segmentation of the skeleton or the parts based on that skeleton is performed, which can include postprocessing like smoothing the skeleton or fitting a B-Spline (Bremicker et al., 1991; Kresslein et al., 2018; Nana et al., 2017; Stangl and Wartzack, 2015). Finally, the cross-sections are determined so that the 3D geometry can be swept along the skeleton by including the cross-sections (Goyal et al., 2012).

In the recent publications of (Nana et al., 2017; Stangl and Wartzack, 2015), the beamline is approximated by a contraction method, which is presented in (Tagliasacchi et al., 2012). Contraction methods act on the polygon mesh and iteratively push the boundary until the surfaces intersect each other (Sobiecki et al., 2013). This can result in a 1D curve or 1D/2D curve/surface skeletons. The contraction method is only applied to the boundary of the geometry, whereas thinning methods erode a volumetric representation. The authors in (Dey et al., 2003; Mayer and Wartzack, 2020; Ramanathan and Gurumoorthy, 2010) use the medial axis transformation directly for the estimation of curve/surface skeletons, which can -in contrast- to the contraction method of (Tagliasacchi et al., 2012), fully cover the original shape (Tagliasacchi et al., 2016). These skeletons often cover the noise of the boundary so that pre-and post-processing steps are often required (Tagliasacchi et al., 2016). The authors of (Bremicker et al., 1991; Denk et al., 2020; Yin et al., 2020) use a thinning-based approach for the calculation of the curve skeleton for images, which can preserve topological properties during erosion. 
While the authors (Dey et al., 2003; Mayer and Wartzack, 2020; Ramanathan and Gurumoorthy, 2010) of being restricted to curve skeletons, the authors of (Dey et al., 2003; Hisada et al., 2001; Mayer and Wartzack, 2020; Ramanathan and Gurumoorthy, 2010; Yin et al., 2020) apply to surface skeletonization but without further processing. We proceed on the surface skeleton using subdivision surfaces similar (Denk et al., 2020), but in contrast to (Denk et al., 2020), we apply it for surface skeleton instead of curve skeletons representing mid-surface. We use the homotopic thinning method of (Lee et al., 1994) to determine the binary surface skeleton and ensure topology preservation in the skeleton itself. The thickness of the segmented skeleton surface is approximated by applying the distance transformation on the binary image. For each surface region, we determine an average thickness to match the subdivision surface control grid. Similar to (Hisada et al., 2001), we use the edges and the junctions of the surface skeleton, estimating the parametrization.

\section{SKELETON BASED SUBDIVISION SURFACE ESTIMATION}

A mid-surface parametrization can be defined by the estimation of the mid surface and the corresponding thickness. Therefore, our parametrization should cover similar behavior. The reconstruction in this article of topology optimized results into a surface parametrization is covered in Figure 3.

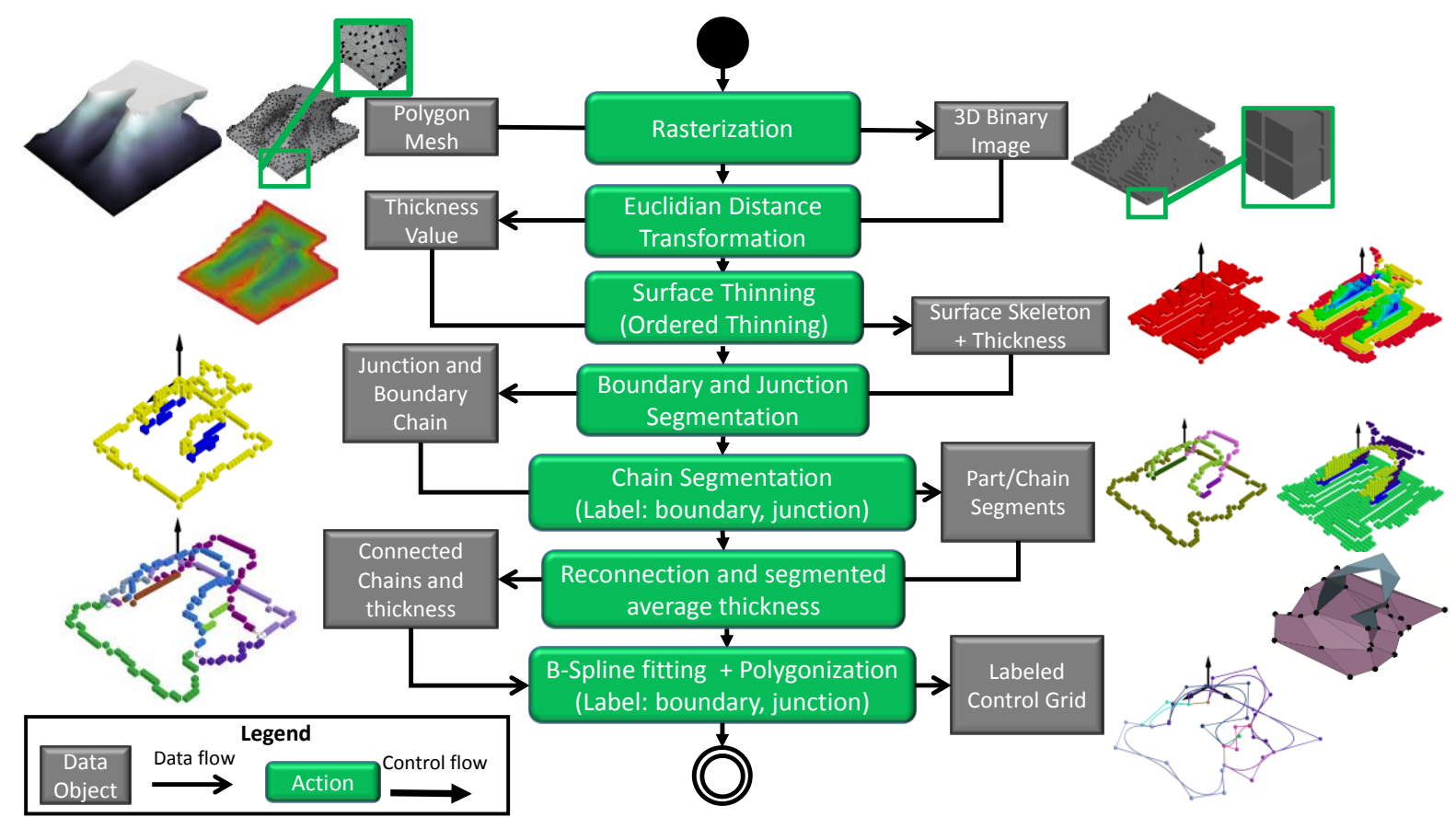

Figure 3: Automated segmented subdivision surface control grid with an individual thickness

First, the polygon mesh is rasterized. Second, the modified surface skeletonization of (Lee et al., 1994) using a distance transformation ordered criteria is applied. This leads to a binary skeleton. Based on the Euclidian distance transformation on the binary image, each skeleton point covers a distance value. These distance values serve as an approximation for the thickness of each corresponding part. Third, the junction line and the boundary of the digital surface are selected, which serves as the boundary in the parametrization. Fifth, the junction line is extended until all corresponding parts are connected. Sixth, a B-Spline interpolation of each independent spline segment serves for the control points of the subdivision surface parametrization. Based on this control grid and the corresponding thickness value, the control points can be extruded to solid geometry. The following figure shows the desirable surface parametrization of a polygon mesh.

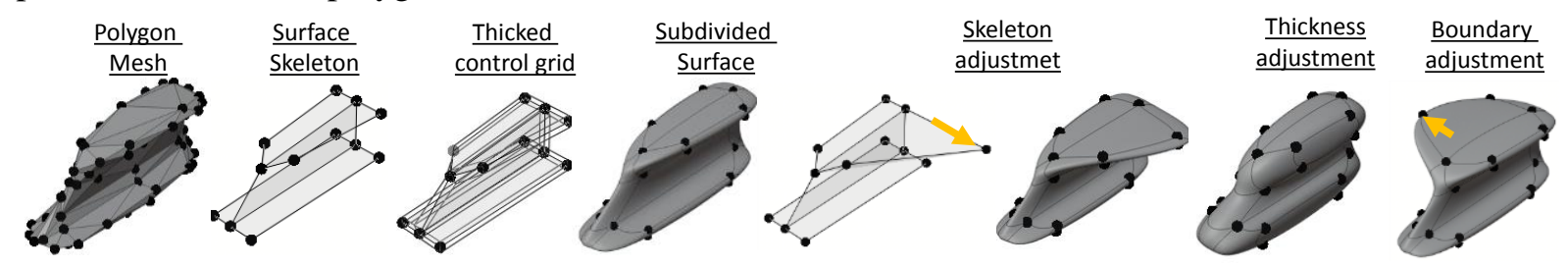

Figure 4: Surface skeleton subdivision surface parametrization 
The shape is defined by a surface skeleton, which can be ticked to a control grid for subdivision surfaces. Each surface on the control grid is defined with a constant thickness. Based on that parametrization, the thickness, the skeleton, and the ticked control grid can be adjusted. Such flexible shape description can be used for further shape optimizations (Bandara et al., 2016). These have to be restricted so that the topology does not change (Storti et al., 1997), and the shape does fulfill the Jordan curve theorem.

While surface skeleton can cover arbitrary shapes (Tagliasacchi et al., 2016), the used mid-surface parametrization is restricted to a constant local thickness for each patch. So, a thickness change is covered by segmenting the skeleton into several surface patches.

\subsection{Ordered Surface skeletonization}

A binary digital image $V=\mathbb{Z}^{3}$ is an image consisting of black voxels $B \subseteq V$ and white voxels $V \backslash B$. Due to the thinning of $B$, voxels $v(x, y, z)$ on the surface of $B$ will be removed until a one thick voxel representation of curves and surfaces is available. To achieve a suitable thinning process, the erosion $B \ominus S$ with a structure element $S$ has to be restricted. Therefore, a so-called simple point condition can be used (Kong and Rosenfeld, 1989; Lee et al., 1994; Morgenthaler, 1981), by which only simple points $v$ on the surface are deleted which do not alter the topological properties such as number of holes, number of cavities and number of objects. In combination with an end point criteria, a topology preserving and shape preserving thinning method can be constructed (Kong and Rosenfeld, 1989; Lee et al., 1994). For the detection of the surface skeleton, we use a modified version of the thinning method of (Lee et al., 1994) by adding an ordered distance approach similar to (Siddiqi et al., 2002) based on the Euclidian distance transformation. Figure 5 covers the skeletonization method and explains this method by using a $2 \mathrm{D}$ example, whereas the grey pixel determines the candidates for erosion. For ordered thinning local density in topology optimization or singal strenght in CT-Data can be choosen.

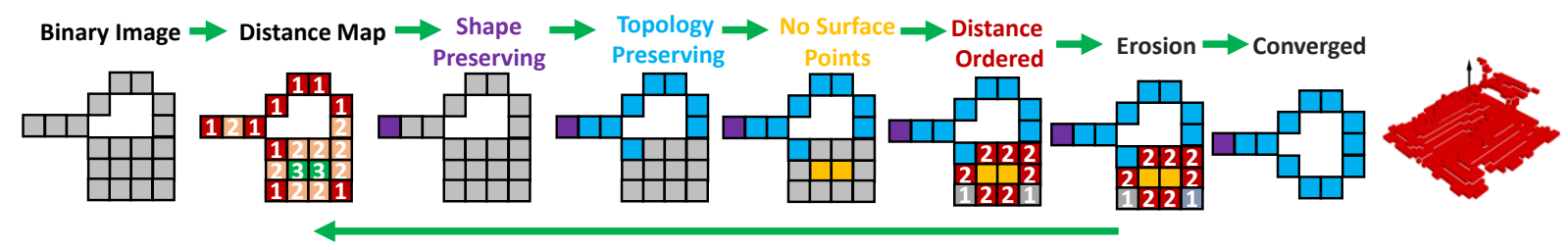

Figure 5: Homotopic distance ordered thinning on a $3 D$ and 2D image

Firstly, the distance of the binary image is approximated. Secondly, the distance transformation is applied to that binary image. Thirdly, a shape condition preserving the endpoints of $1 \mathrm{D}$ curves and surfaces is used similarly to (Lee et al., 1994). Fourth, simple points are detected, which do not alter the topology and the shape-preserving condition after removal. Fifth, only pixels or voxels on the surface are selected. Sixth, these candidates are ordered by their distance, whereas only the candidates with the smallest value are selected. Seventh, one of the candidates is eroded. This procedure is repeated until convergence so that only a 1D-pixel thick line or surface geometry is preserved. Additionally to the procedure, an example of a 2D surface skeleton is visualized. This example shows several different point types (junction, boundary, ...), which are used in the following section for a reasonable segmentation.

\subsection{Part and Skeleton Segmentation}

The boundary of the surface skeleton is extracted by the defined patterns in endpoint criteria for surfaces covered in (Lee et al., 1994). Junction points can be approximated if the number of the surrounding voxels $N_{26}(v)$ exceeds 8 . These lines are thinned to 1D thick curves, including junction line. For part segmentation, each chain (no junction chain) is growing until convergence. Afterward, the missing connections on the junction chains are reconnected depending on the corresponding surrounding parts. First, the boundary line (yellow) and the junction lines on the surface skeleton are extracted. Second, these lines are thinned so that only one thick line curve results. The lines with a thickness of one voxel are segmented into several chains separating junction and boundary and including branch points if available. Third, based on these chains, the segmentation of the surface skeleton is covered by region growing of all non-junction chains, whereas the junction line serves as a stop criterion for growing. The average thickness of each segment is stored for the thickening of each component. Fourth, on each junction point, the missing connection is added based on the part segmentation. The estimation of the 
control grid was covered by fitting B-Splines using the method described in (Dierckx, 1982). The following figure shows the transformation of voxel lines into a segmented polygon model.

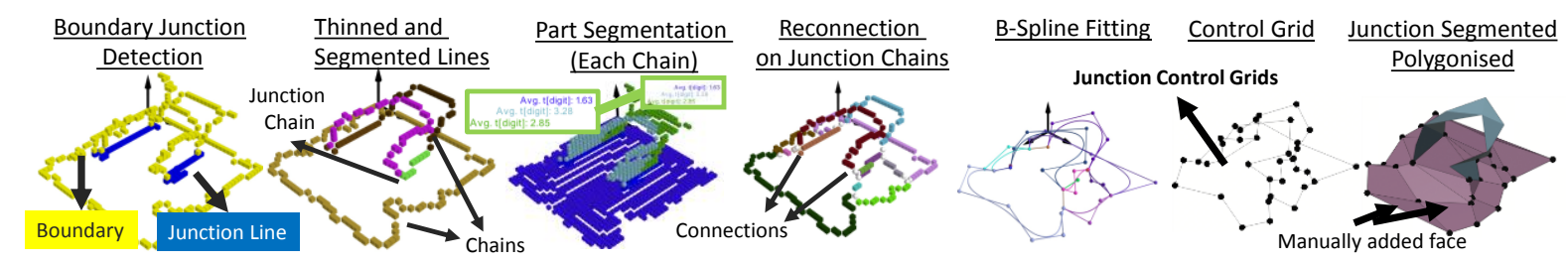

Figure 6: Voxel lines to the polygon control grid

The labeled chains are fitted to B-Splines, whereas the control points of the B-Splines serve as the vertices for the polygon model. Then, the control grid is fitted on the control points labeled as a junction. Each disconnected control grid set is polygonized with triangles and quads. In this case, some triangles are manually added to the purple model, represents a smooth boundary. The following section covers the subdivision surface parametrization and the shape adjustment in more detail. So, the proposed approach automatically determines a voxel surface skeleton, which is automatically converted into a mid-surface shape consisting of control grids for the subdivision surfaces. To generate a subdivision surface 3D model, the steps, as shown in Figure 7, are applied to the 2D segmented polygon mesh.

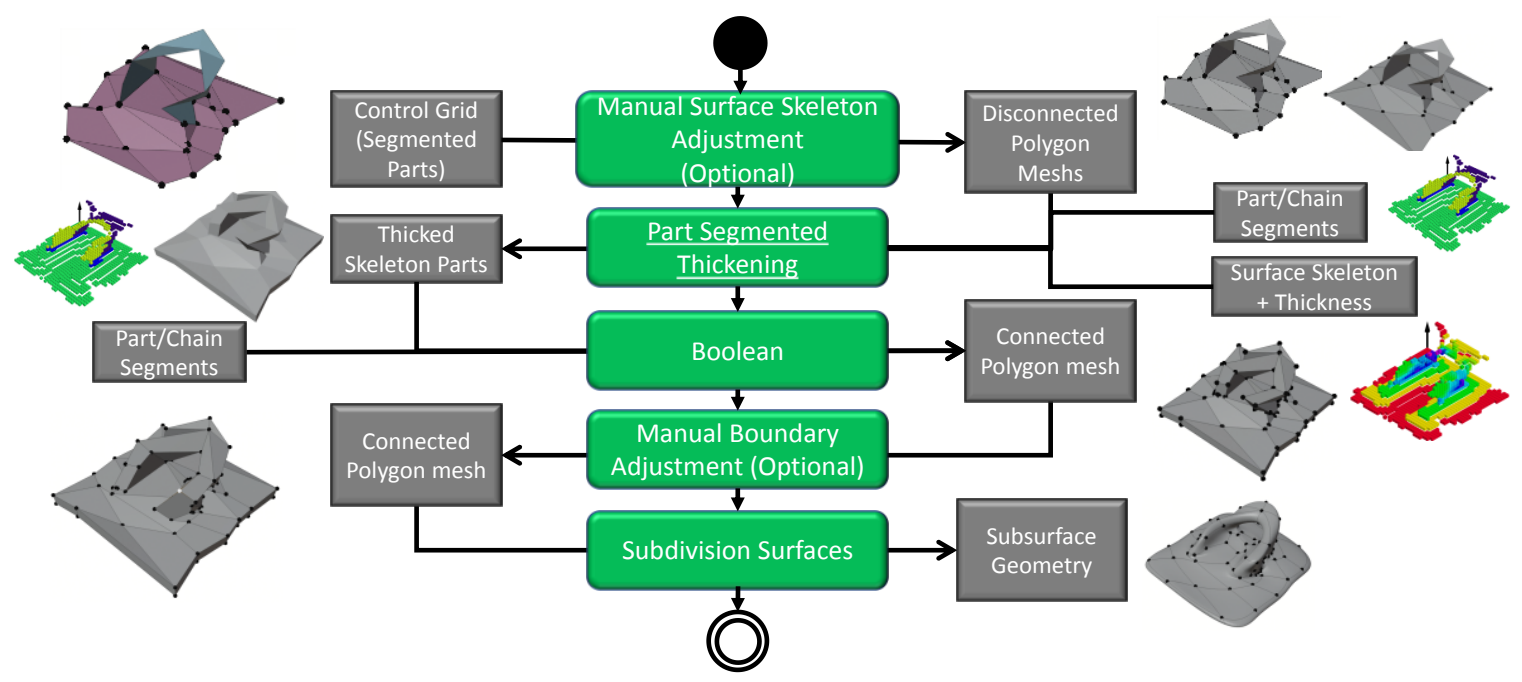

Figure 7: Control grid adjustment and solid subdivision surface geometry.

First, the surface polygon skeleton can be adjusted so that some parts fit better to the original geometry. Second, based on the part/chain segments and the thickness of the segmented polygon meshes, the polygon mesh can be thickened to a 3D geometry. Third, each independent thickened part is then unified into one polygon model (surface model). Fourth, on that surface model, manual adjustment can be applied. The resulting control grid can now be subdivided until a smooth geometry will result.

\section{SUBDIVISION SURFACE PARAMETRIZATION OF SEVERAL USE-CASES}

The experimental part is divided into two sections. First, several different examples where at first, the control grid is automatically determined, then subsequently, the subdivision surfaces are generated based on what is covered. The samples are generated using different design spaces and load conditions. Most of the selected parts mismatch the recommended shape of a constant local thickness so that certain behaviors can be shown. Second, shape adjustment is applied with the subdivision surface parametrization. 
Table 1: Subdivision surface control grid estimation on several use-cases

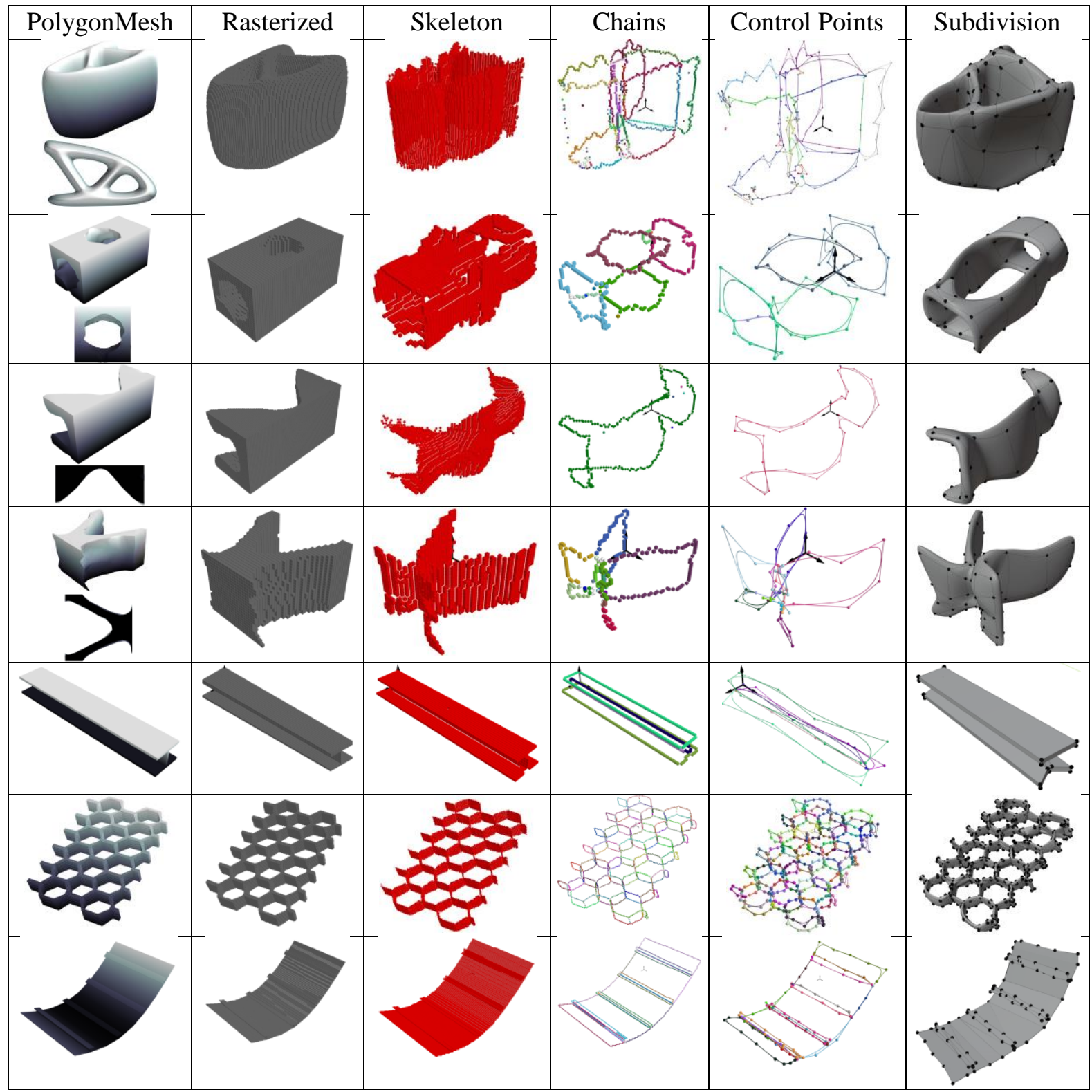

The control grid estimation using the surface skeleton leads to promising results presented in Table 1, but in contrast to the curve skeleton in Figure 1, the number of control points increases dramatically, but the surface skeleton can cover non-beam-like shapes. The following figure shows the adjustment of the mid-surface and the smooth connection provided by the ticked subdivision surface grid. Due to the smooth subdivision surface, the sharp edges of the honeycomb are smoothed.

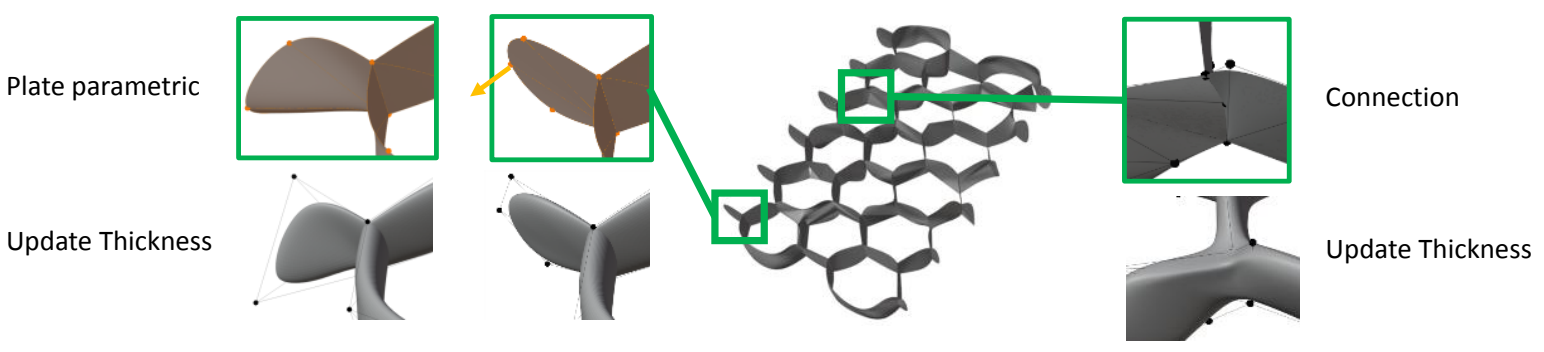

Figure 8: Update of the mid-surface parametric and visualization of one junction

Adjusting the shape until the original geometry is reconstructed is dealt with in the following figure using the surface parametrization and the boundary representation (thicked). Changing the surface parametrization results in a huge deformation change. For parts with a thickness variety, the boundary has to be changed but changing the boundary requires more action due to the double number of vertices. 

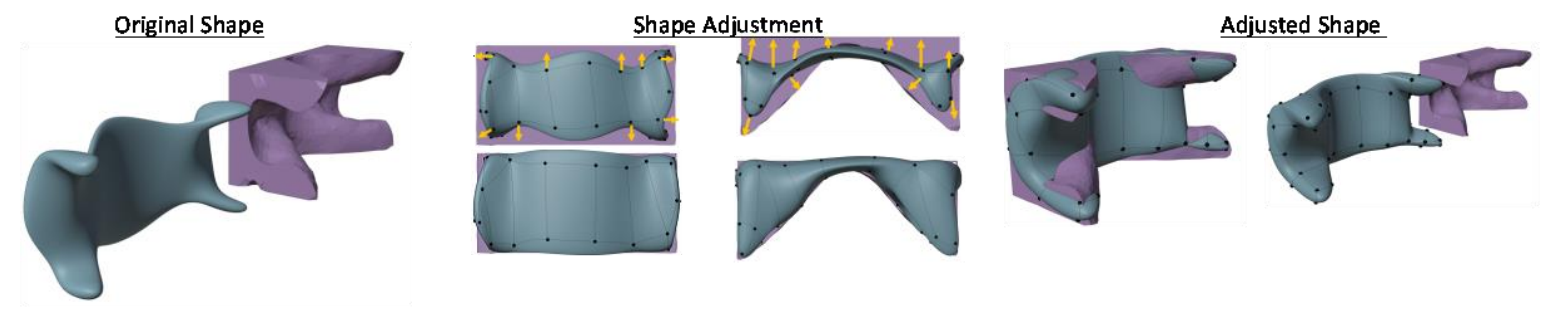

Figure 9: Shape adjustment

To enable a reasonable parametrization, the proposed procedure should be restricted to organic shapes due to the smoothing of the subdivision surface procedure, and it has to be ensured that small details are covered during the rasterization. Additionally, each thickness of a surface segment provided by part segmentation should be approximately the same. Furthermore, the designer has always to consider that every contour is approximated by a skeleton so that a circular hole will be represented as a thicked circular instead of subtracting a cylinder using CSG.

\section{CONCLUSION AND SUMMARY}

This article presents a parameterization of polygon meshes into a parametric representation using subdivision surfaces. For this purpose, we estimate a surface skeleton determined by an ordered homotopic thinning method and analyze the skeleton using the local neighborhood of each voxel. Based on that voxel-skeleton, we extract voxel-chains and several independent parts (part segmentation), which represent the main shape of the geometry. For each chain, reasonable control points are estimated by fitting B-Splines. By additionally adding thickness values, a 3D control grid can be enabled. Based on that method, the designer can choose an existing geometry that will be converted in such representation. This representation can be manually modified using computer graphic software or imported in CAD tools for industrial design. Additionally, due to the erosion of the geometry, the resulting $2 \mathrm{D}$ skeleton is shifted inside the geometry. Therefore, the control grid has to be pushed back to the boundary for some instances, as visualized in Figure 9. The voxel-surface-skeleton itself, as shown in Table 1, is noisy so that for further research, and additional pre-processing step should be applied, or the skeletonization method for digital images itself should be switched to methods which smoother results similar to (Couprie and Bertrand, 2015). The digital space itself offers advantages for robust part-segmentation, chain-segmentation, preserving the topology, and including volumetric properties such as the boundary distance. The control grid estimation leads to promising results, whereas most of the topology and shape are covered in the resulting 3D geometry, as visualized in Table 1. Currently, the individual parts are segmented only due to the junction lines, which do not cover geometry changes. For further research, the local thickness and the change of the skeleton (curvature, normal vectors) should also be considered segmenting the mid-surface. Additionally, the surface control grid can be sharpened by detecting sharp edges using a feature line detection method similar to (Denk et al., 2019).

\section{ACKNOWLEDGMENT}

This work is part of the project ANGORA, which is supported by the "Federal Ministry for Economic Affairs and Energy" with the funding indicator ZF4428401BZ7 in the context of the research network IraSME and ZIM. Responsibility for the content of this publication lies with the authors.

\section{REFERENCES}

Adam, G.A.O., Zimmer, D., 2015. On design for additive manufacturing: evaluating geometrical limitations. Rapid Prototyp. J. 21, 662-670. https://doi.org/10.1108/RPJ-06-2013-0060

Agathos, A., Pratikakis, I., Perantonis, S., Sapidis, N., Azariadis, P., 2007. 3D Mesh Segmentation Methodologies for CAD applications. Comput.-Aided Des. Appl. 4, 827-841. https://doi.org/10.1080/16864360.2007.10738515

Bandara, K., Rüberg, T., Cirak, F., 2016. Shape optimisation with multiresolution subdivision surfaces and immersed finite elements. Comput. Methods Appl. Mech. Eng. 300, 510-539. https://doi.org/10.1016/j.cma.2015.11.015

Ben Makhlouf, A., Louhichi, B., Mahjoub, M.A., Deneux, D., 2019. Reconstruction of a CAD model from the deformed mesh using B-spline surfaces. Int. J. Comput. Integr. Manuf. 32, 669-681.

https://doi.org/10.1080/0951192X.2019.1599442 
Bendsøe, M.P., Sigmund, O., 1999. Material interpolation schemes in topology optimization. Arch. Appl. Mech. 69, 635-654. https://doi.org/10.1007/s004190050248

Bénière, R., Subsol, G., Gesquière, G., Le Breton, F., Puech, W., 2013. A comprehensive process of reverse engineering from 3D meshes to CAD models. Comput.-Aided Des. 45, 1382-1393. https://doi.org/10.1016/j.cad.2013.06.004

Blum, H., 1967. A Transformation for Extracting New Descriptors of Shape. M.I.T. Press.

Bremicker, M., Chirehdast, M., Kikuchi, N., Papalambros, P.Y., 1991. Integrated Topology and Shape Optimization in Structural Design*. Mech. Struct. Mach. 19, 551-587. https://doi.org/10.1080/08905459108905156

Couprie, M., Bertrand, G., 2015. A 3D Sequential Thinning Scheme Based on Critical Kernels, in: Benediktsson, J.A., Chanussot, J., Najman, L., Talbot, H. (Eds.), Mathematical Morphology and Its Applications to Signal and Image Processing, Lecture Notes in Computer Science. Springer International Publishing, pp. 549-560.

Dedè, L., Borden, M.J., Hughes, T.J.R., 2012. Isogeometric Analysis for Topology Optimization with a Phase Field Model. Arch. Comput. Methods Eng. 19, 427-465. https://doi.org/10.1007/s11831-012-9075-z

Denk, M., Paetzold, K., Rother, K., 2019. Feature line detection of noisy triangulated CSGbased objects using deep learning, in: Proceedings of the 30th Symposium Design for X (DFX 2019), DfX. Presented at the DfX Symposium 2019, The Design Society, Jesteburg, Germany, pp. 239-250. https://doi.org/10.35199/dfx2019.21

Denk, M., Rother, K., Paetzold, K., 2020a. Fully Automated Subdivision Surface Parametrization for Topology Optimized Structures and Frame Structures using Euclidean Distance Transformation and Homotopic Thinning, in: Proceedings of the Munich Symposium on Lightweight Design 2020. Springer Nature, Munich, Germany. https://doi.org/10.1007/978-3-662-63143-0

Denk, M., Rother, K., Paetzold, K., 2020b. Multi-Objective Topology Optimization of Heat Conduction and Linear Elastostatic using Weighted Global Criteria Method, in: Proceedings of the 31st Symposium Design for X (DFX2020), DFX. Presented at the DfX Symposium 2020, The Design Society, Bamberg, pp. 91100. https://doi.org/10.35199/dfx2020.10

Dey, T.K., Woo, H., Zhao, W., 2003. Approximate medial axis for CAD models, in: Proceedings of the Eighth ACM Symposium on Solid Modeling and Applications, SM '03. Association for Computing Machinery, New York, NY, USA, pp. 280-285. https://doi.org/10.1145/781606.781652

Dierckx, P., 1982. Algorithms for smoothing data with periodic and parametric splines. Comput. Graph. Image Process. 20, 171-184. https://doi.org/10.1016/0146-664X(82)90043-0

Feng, C., Jalba, A.C., Telea, A.C., 2015. Part-Based Segmentation by Skeleton Cut Space Analysis, in: Benediktsson, J.A., Chanussot, J., Najman, L., Talbot, H. (Eds.), Mathematical Morphology and Its Applications to Signal and Image Processing, Lecture Notes in Computer Science. Springer International Publishing, Cham, pp. 607-618. https://doi.org/10.1007/978-3-319-18720-4_51

Gao, J., Gao, L., Luo, Z., Li, P., 2019. Isogeometric topology optimization for continuum structures using density distribution function. Int. J. Numer. Methods Eng. 119, 991-1017. https://doi.org/10.1002/nme.6081

Gao, J., Luo, Z., Xiao, M., Gao, L., Li, P., 2020. A NURBS-based Multi-Material Interpolation (N-MMI) for isogeometric topology optimization of structures. Appl. Math. Model. 81, 818-843. https://doi.org/10.1016/j.apm.2020.01.006

Gauthier, S., Puech, W., Bénière, R., Subsol, G., 2017. Analysis of digitized 3D mesh curvature histograms for reverse engineering. Comput. Ind. 92-93, 67-83. https://doi.org/10.1016/j.compind.2017.06.008

Goyal, M., Murugappan, S., Piya, C., Benjamin, W., Fang, Y., Liu, M., Ramani, K., 2012. Towards locally and globally shape-aware reverse 3D modeling. Comput.-Aided Des. 44, 537-553. https://doi.org/10.1016/j.cad.2011.12.004

Hisada, M., Belyaev, A.G., Kunii, T.L., 2001. A 3D Voronoi-based skeleton and associated surface features, in: Proceedings Ninth Pacific Conference on Computer Graphics and Applications. Pacific Graphics 2001, pp. 89-96. https://doi.org/10.1109/PCCGA.2001.962861

Hua Li, Yezzi, A., 2006. Vessels as 4D Curves: Global Minimal 4D Paths to Extract 3D Tubular Surfaces, in: 2006 Conference on Computer Vision and Pattern Recognition Workshop (CVPRW'06), pp. 82-82. https://doi.org/10.1109/CVPRW.2006.210

Koehoorn, J., Feng, C., Kustra, J., Jalba, A., Telea, A., 2017. Unified part-patch segmentation of mesh shapes using surface skeletons, in: Skeletonization. Elsevier, Netherlands, pp. 89-122. https://doi.org/10.1016/B978-0-08-101291-8.00005-5

Kong, T.Y., Rosenfeld, A., 1989. Digital topology: Introduction and survey. Comput. Vis. Graph. Image Process. 48, 357-393. https://doi.org/10.1016/0734-189X(89)90147-3

Kresslein, J., Haghighi, P., Park, J., Ramnath, S., Sutradhar, A., Shah, J.J., 2018. Automated cross-sectional shape recovery of 3D branching structures from point cloud. J. Comput. Des. Eng. 5, 368-378. https://doi.org/10.1016/j.jcde.2017.11.010

Lee, T.C., Kashyap, R.L., Chu, C.N., 1994. Building Skeleton Models via 3-D Medial Surface Axis Thinning Algorithms. CVGIP Graph. Models Image Process. 56, 462-478. https://doi.org/10.1006/cgip.1994.1042

Lian, H., Christiansen, A.N., Tortorelli, D.A., Sigmund, O., Aage, N., 2017. Combined shape and topology optimization for minimization of maximal von Mises stress. Struct. Multidiscip. Optim. 55, 1541-1557. https://doi.org/10.1007/s00158-017-1656-x 
Lidayová, K., Frimmel, H., Wang, C., Bengtsson, E., Smedby, Ö., 2016. Fast vascular skeleton extraction algorithm. Pattern Recognit. Lett., Special Issue on Skeletonization and its Application 76, 67-75. https://doi.org/10.1016/j.patrec.2015.06.024

Louhichi, B., Abenhaim, G.N., Tahan, A.S., 2015. CAD/CAE integration: updating the CAD model after a FEM analysis. Int. J. Adv. Manuf. Technol. 76, 391-400. https://doi.org/10.1007/s00170-014-6248-y

Mayer, J., Wartzack, S., 2020. Ermittlung eines Skelettierungsverfahrens zur Konvertierung von Topologieoptimierungsergebnissen, in: Proceedings of the 31st Symposium Design for X (DFX2020). Presented at the Symposium Design for X 2020, Bamberg, pp. 111-120. https://doi.org/10.35199/dfx2020.12

Morgenthaler, D.G., 1981. Three-dimensional Simple Points: Serial Erosion, Parallel Thinning, and Skeletonization. University of Maryland.

Nana, A., Cuillière, J.-C., Francois, V., 2017. Automatic reconstruction of beam structures from 3D topology optimization results. Comput. Struct. 189, 62-82. https://doi.org/10.1016/j.compstruc.2017.04.018

Picelli, R., Townsend, S., Brampton, C., Norato, J., Kim, H.A., 2018. Stress-based shape and topology optimization with the level set method. Comput. Methods Appl. Mech. Eng. 329, 1-23. https://doi.org/10.1016/j.cma.2017.09.001

Ramanathan, M., Gurumoorthy, B., 2010. Interior Medial Axis Transform computation of 3D objects bound by free-form surfaces. Comput.-Aided Des. 42, 1217-1231. https://doi.org/10.1016/j.cad.2010.08.006

Reniers, D., Telea, A., 2008a. Part-type Segmentation of Articulated Voxel-Shapes using the Junction Rule. Comput. Graph. Forum 27, 1845-1852. https://doi.org/10.1111/j.1467-8659.2008.01331.x

Reniers, D., Telea, A., 2008b. Patch-type Segmentation of Voxel Shapes using Simplified Surface Skeletons. Comput. Graph. Forum 27, 1837-1844. https://doi.org/10.1111/j.1467-8659.2008.01330.x

Saha, P.K., Gomberg, B.R., Wehrli, F.W., 2000. Three-dimensional digital topological characterization of cancellous bone architecture. Int. J. Imaging Syst. Technol. 11, 81-90. https://doi.org/10.1002/(SICI)10981098(2000)11:1\&lt;81::AID-IMA9>3.0.CO;2-1

Siddiqi, K., Bouix, S., Tannenbaum, A., Zucker, S.W., 2002. Hamilton-Jacobi Skeletons. Int. J. Comput. Vis. 48, 215-231. https://doi.org/10.1023/A:1016376116653

Sobiecki, A., Yasan, H.C., Jalba, A.C., Telea, A.C., 2013. Qualitative Comparison of Contraction-Based Curve Skeletonization Methods, in: Hendriks, C.L.L., Borgefors, G., Strand, R. (Eds.), Mathematical Morphology and Its Applications to Signal and Image Processing, Lecture Notes in Computer Science. Springer Berlin Heidelberg, pp. 425-439.

Stangl, T., Wartzack, S., 2015. Feature based interpretation and reconstruction of structural topology optimization results, in: Weber, M., C.;. Husung, S.;. Cascini, G.;. Cantamessa, M.;. Marjanovic, D.;. Bordegoni (Ed.), Proceedings of the 20th International Conference on Engineering Design (ICED15). Design Society, p. Vol. 6, 235-245.

Storti, D.W., Turkiyyah, G.M., Ganter, M.A., Lim, C.T., Stal, D.M., 1997. Skeleton-based modeling operations on solids, in: Proceedings of the Fourth ACM Symposium on Solid Modeling and Applications, SMA '97. Association for Computing Machinery, New York, NY, USA, pp. 141-154. https://doi.org/10.1145/267734.267771

Svensson, S., Nyström, I., Sanniti di Baja, G., 2002. Curve skeletonization of surface-like objects in 3D images guided by voxel classification. Pattern Recognit. Lett. 23, 1419-1426. https://doi.org/10.1016/S01678655(02)00102-2

Tagliasacchi, A., Alhashim, I., Olson, M., Zhang, H., 2012. Mean Curvature Skeletons. Comput. Graph. Forum 31, 1735-1744. https://doi.org/10.1111/j.1467-8659.2012.03178.x

Tagliasacchi, A., Delame, T., Spagnuolo, M., Amenta, N., Telea, A., 2016. 3D Skeletons: A State-of-the-Art Report. Comput. Graph. Forum 35, 573-597. https://doi.org/10.1111/cgf.12865

Vidal, V., Wolf, C., Dupont, F., 2014. Mechanical Mesh Segmentation and Global 3D Shape Extraction.

Wang, P., Gan, Y., Shui, P., Yu, F., Zhang, Y., Chen, S., Sun, Z., 2018. 3D shape segmentation via shape fully convolutional networks. Comput. Graph. 76, 182-192. https://doi.org/10.1016/j.cag.2018.07.011

Weber, S., Montero, J., Bleckmann, M., Paetzold, K., 2020. Parameters on Support Structure Design for Metal Additive Manufacturing. Proc. Des. Soc. Des. Conf. 1, 1145-1154. https://doi.org/10.1017/dsd.2020.14

Wu, B., Xu, K., Zhou, Y., Xiong, Y., Huang, H., 2016. Skeleton-guided 3D shape distance field metamorphosis. Graph. Models, SI: CVM 2016 selected Papers 85, 37-45. https://doi.org/10.1016/j.gmod.2016.03.003

Yin, G., Xiao, X., Cirak, F., 2020. Topologically robust CAD model generation for structural optimisation. Comput. Methods Appl. Mech. Eng. 369, 113102. https://doi.org/10.1016/j.cma.2020.113102

Yoely, Y.M., Amir, O., Hanniel, I., 2018. Topology and shape optimization with explicit geometric constraints using a spline-based representation and a fixed grid. Procedia Manuf., 15th Global Conference on Sustainable Manufacturing 21, 189-196. https://doi.org/10.1016/j.promfg.2018.02.110 